\title{
Microcavity Brillouin Laser with High Coherence
}

\author{
Jiang Li, Hansuek Lee, Tong Chen, Oskar Painter, Kerry Vahala \\ Thomas J. Watson, Sr., Laboratory of Applied Physics, California Institute of Technology, \\ 1200 E. California Boulevard, Pasadena, CA 91125, USA \\ vahala@caltech.edu
}

Abstract: The first chip-based stimulated Brillouin laser (SBL) is demonstrated. It has efficiency of $90 \%$ and exhibits record coherence for an on-chip device, featuring Schawlow-Townes frequency noise of $60 \mathrm{milliHz}^{2} / \mathrm{Hz}$. Low technical noise is also observed.

OCIS codes: (190.5890) Stimulated; (290.5900) stimulated Brillouin; (190.4390) Integrated optics.

\section{Introduction}

High coherence lasers (very low frequency noise) are critically important for high-performance microwave oscillators [1]; coherent fiber-optic communications [2]; remote sensing [3]; and in atomic physics [4]. In these applications, the twin goals of miniaturization and attainment of high coherence prove very challenging, because quantum noise and technical noise contributions to laser coherence typically increase as laser-cavity form factor is decreased [5]. In this work, we demonstrate a stimulated Brillouin laser (SBL) that attains the highest level of coherence reported for a chip-based device. Schawlow-Townes noise of $60 \mathrm{milliHz} / \mathrm{Hz}$ is measured at an output power of approximately $400 \mu \mathrm{W}$, and low-frequency technical noise is comparable to commercial fiber lasers. The devices are also the first chip-based SBLs and leverage a new ultra-high-Q wedge cavity that enables precise matching of the Brillouin gain shift to the free-spectral-range (FSR), thereby guaranteeing oscillation. More than $90 \%$ conversion of lower-coherence pump to high-coherence output is possible, and threshold powers around 60 microWatts are measured.

\section{Matching of FSR to Brillouin Shift}

While stimulated Brillouin scattering is a well-known effect, its application in microcavities for laser sources has only recently been documented [6,7]. The principle challenge is to create sufficiently high-Q devices with the requisite mode spacing so as to precisely frequency match the narrow-band Brillouin gain spectrum. So far, this matching process has been somewhat statistical in nature, relying upon variation in resonator size and shape to create accidental matches. In this work, we apply a new process for fabricating resonators of precisely controlled diameter with $\mathrm{Q}$ factors as high as 870 million [8]. Using only lithography and a buffered hydrofluoric etch, wedgeshaped resonators are created as shown in the top left inset of Fig. 1. The diameter of these devices can be controlled to sub micron precision making possible an exact frequency match to the Brillouin process. In the measurement, the resonator is pumped on resonance through a fiber taper coupler [9] and Brillouin oscillation occurs in the opposite direction. The threshold for the process is typically in the range of 60-100 microWatts. By adjusting the cavity loading so as to critically couple the pump at the desired laser output power, $95 \%$ differential efficiency is achieved with output power more than $1 \mathrm{~mW}$, as shown in Fig. 1.

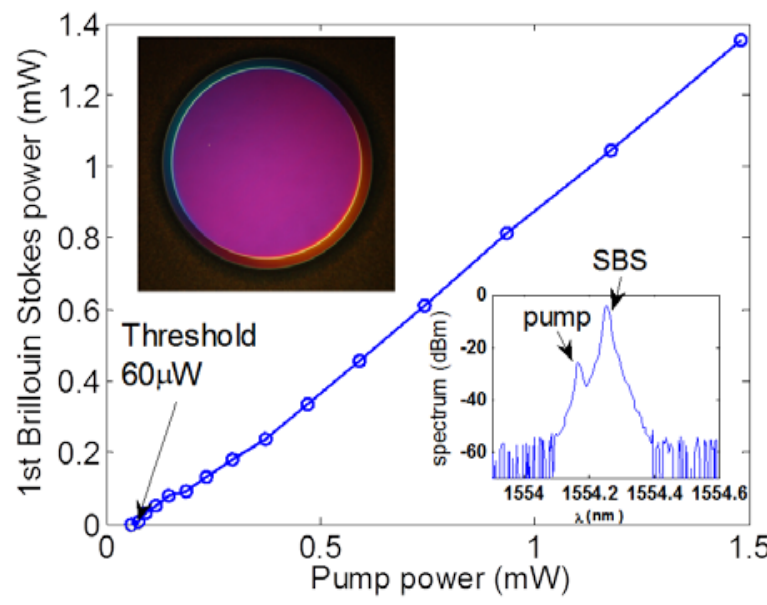

Fig. 1. Main panel: Output power of the first Brillouin Stokes laser line versus coupled optical pump power. Insets: (lower) Optical spectrum showing the Stokes line and the pump wave. Pump is suppressed since laser output power propagates opposite to the direction of pumping. (upper) Micrograph of disk resonator.

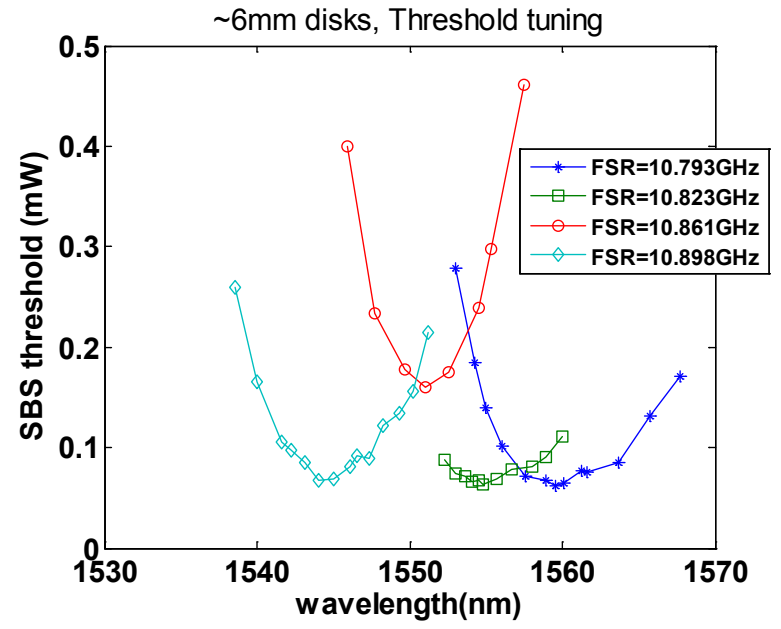

Fig. 2. Measured threshold versus pump detuning on four resonators, each of slightly different diameter (and hence FSR). The minima correspond to matching of the FSR with the Brillouin gain maximum. Tuning of the pump wave away from the maximum causes mismatch and hence an increase in threshold. 
The precise control of microcavity diameter to achieve a frequency match with the Brillouin process is illustrated in Fig. 2 where four resonator diameters (5970, 5994, 6012 and 6030 microns) were tested at a series of pump wavelengths in the $1550 \mathrm{~nm}$ band. The minimum threshold corresponds to excitation at the Brillouin gain maximum. The rise in threshold away from the minimum (for a given resonator diameter) reflects slight tuning of the Brillouin gain-peak frequency shift with pump wavelength change due to photon-phonon momentum conservation.

\section{Phase Noise Characterization of High Coherence Laser}

The phase noise of these devices has been characterized in several ways. A Mach-Zehnder interferometer is used to measure the SBL frequency noise from around $0.1 \mathrm{MHz}$ to $10 \mathrm{MHz}$. Above $1 \mathrm{MHz}$ frequency offset, the device is quantum limited. The measured Schawlow-Townes noise is plotted in Fig. 3 and features a characteristic inversepower dependence. At 375 micoWatts of output power, the measured Schawlow-Townes frequency noise is 60 milliHz $/ \mathrm{Hz}$, which is the lowest level ever reported for a chip-based device. The use of fiber-based SBLs to generate coherent microwaves has also been reported [10]. By operating the current device in cascade we can generate a coherent microwave signal by photomixing the Stokes lines together on a high-speed photodetector. The Fig. 4 inset shows that a narrow beat note is observed between the second and third order SBS stokes lines at 10.889GHz. The phase noise of the microwave beat note is measured on a phase noise analyzer and is plotted in Fig. 4. This phase noise spectrum permits analysis of residual technical noise contributions to the SBL and will be discussed.

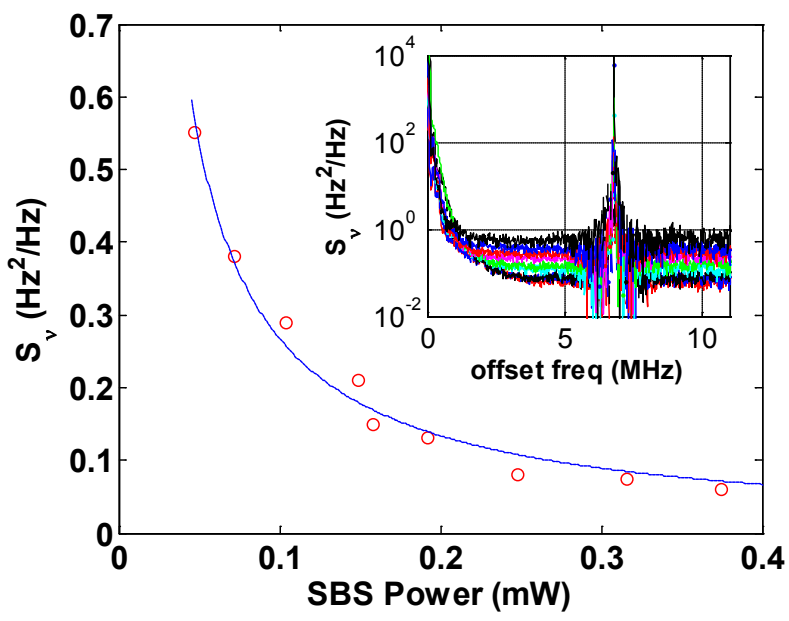

Fig. 3. Main panel: Measured Schawlow-Townes noise versus laser output power. A minimum noise level of $60 \mathrm{milliHz}^{2} / \mathrm{Hz}$ is obtained at 375 microWatts. Inset: Frequency fluctuation spectrum $S_{v}$ at different output powers. The peak at $6.7 \mathrm{MHz}$ is due to the data conversion from the sinc like transfer function of Mach-Zehnder interferometer.

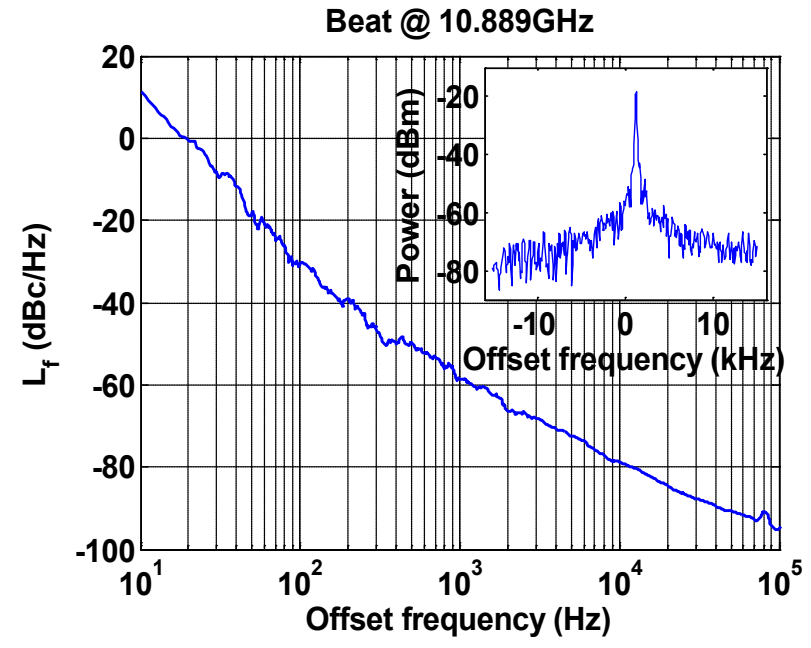

Fig. 4. Main panel: Phase noise of the microwave beat note at $10.889 \mathrm{GHz}$ produced by mixing the 2 nd and 3rd order Stokes lines on a fast photodetector. Inset: Electrical spectrum of the beat note.

\section{References}

[1] T. Fortier, M. Kirchner, F. Quinlan, J. Taylor, J. Berquist, T. Rosenband, N. Lemke, A. Ludlow, Y. Jiang, C. Oates and S. Diddams, "Generation of ultrastable microwaves via optical frequency division", Nature Photonics 5, 425-429 (2011)

[2] E. IP, A. Lau, D. Barros and J. Kahn, "Coherent detection in optical fiber systems", Opt. Exp. 16, 753-791 (2008)

[3] C. Karlsson, F. Olsson, D. Letalick, and M. Harris, "All-fiber multifunction CW coherent laser radar at $1.55 \mu \mathrm{m}$ for range, speed, vibration, and wind measurements, " Appl. Opt. 39, 3716-3726 (2000)

[4] H.S. Margolis, G.P. Barwood, G. Huang, H.A. Klein, S.N. Lea, K. Szymaniec and P. Gill, "Hertz-level measurement of the optical clock frequency in a single ${ }^{88} \mathrm{Sr}^{+}$ion", Science 306, 1355-1358 (2004).

[5] K. Vahala and A. Yariv, "Semiclassical theory of noise in semiconductor lasers-Part I, " IEEE J. Quantum Electron. 19, 1096-1101 (1983)

[6] M. Tomes and T. Carmon, "Photonic Micro-Electromechanical Systems Vibrating at X-band (11-GHz) Rates," Phys. Rev. Lett. 102, 113601 (2009)

[7] I. Grudinin, A. Matsko and L. Maleki, "Brillouin Lasing with a CaF 2 Whispering Gallery Mode Resonator," Phys. Rev. Lett. 102, 043902 (2009)

[8] H. Lee, T. Chen, J. Li, O. Painter and K. Vahala, "Ultra-High-Q Micro-Cavity on a Silicon Chip", Frontiers In Optics, FWS2 (2011)

[9] M. Cai, O. Painter and K. Vahala, "Observation of critical coupling in a fiber taper to silica-microsphere whispering gallery mode system," Phys. Rev. Lett. 85, 74-77, (2000)

[10] M.L. Dennis, R.M. Sova and T.R. Clark, "Dual-wavelength Brillouin fiber laser for microwave frequency generation," Optical Fiber

Communications Conference, OWJ6 (2007) 